\title{
Alteraciones hematológicas en pacientes portadores del virus de Inmunodeficiencia Humana
}

\section{Hematological alterations in patients with human immunodeficiency virus}

Santiago Pacheco Toro. ${ }^{1}$, Sandy Fierro Vasco. ${ }^{2}$, Juan Vega Vasco. ${ }^{3} \&$ Silvana Vega Vasco. ${ }^{4}$

\begin{abstract}
.
DOI: https://doi.org/10.33262/concienciadigital.v3i1.1.1145

Infection with the Human Immunodeficiency Virus, HIV presents with a variety of hematological alterations and can be seen as a complication of the disease itself or linked to treatment. It is characterized by an ineffective erythropoiesis caused by opportunistic infections, malignancies and drugs. In this article we want to determine the most frequent hematological alterations that occur in patients with human immunodeficiency virus treated at the Enrique Garcés Hospital, located in Quito, Ecuador. A simple random sampling was performed for finite universe. The study is of a retrospective descriptive type of a series of cases in the Comprehensive Care Unit for HIVAIDS patients at Enrique Garcés Hospital. The medical records of the patients treated in 2017 were reviewed. A sample of 105 patients was obtained. It was evidenced that the majority (86.7\%) of the cases correspond to the male sex with an average age of 33 years. The prevalence of anemia was $13.3 \%$. The women had a higher prevalence of anemia. Leukopenia was more frequent in the group of patients with CD4 values below 200 cells / $\mu$. It is concluded that the prevalence of anemia was not very high, it prevailed in the female sex with a hypochromic normocytic pattern. Leukopenia was more frequent in the group of patients with CD4 values below 200 cells $/ \mu$. Platelets in most patients were in normal range.
\end{abstract}

Keywords: Anemia, thrombocytopenia, thrombocytosis, VIH

\footnotetext{
${ }^{1}$ Universidad Estatal de Bolívar, enfermería, Guaranda Ecuador, spacheco@ueb.edu.ec

${ }^{2}$ Universidad Estatal de Bolívar, enfermería, Guaranda Ecuador, sfierrov@ ueb.edu.ec

${ }^{3}$ Ministerio de Salud Pública Ecuador, juanmarcelo91@gmail.com

${ }^{4}$ Universidad Católica de Quito, silvanavv89@gmail.com
} 


\section{Resumen.}

La infección por el Virus de la Inmunodeficiencia Humana, VIH se presenta con una variedad de alteraciones hematológicas y pueden verse como complicación de la propia enfermedad o vinculadas al tratamiento. Se caracteriza por una eritropoyesis ineficaz causada por infecciones oportunistas, neoplasias y fármacos. En este artículo se quiere determinar las alteraciones hematológicas más frecuentes que se producen en pacientes portadores del virus de la inmunodeficiencia humana atendidos en el Hospital Enrique Garcés, localizado en Quito, Ecuador. Se realizó un muestreo aleatorio simple para universo finito. El estudio es de tipo descriptivo retrospectivo de una serie de casos en la Unidad de atención integral a pacientes con VIH-SIDA del Hospital Enrique Garcés. Se revisaron las historias clínicas de los pacientes atendidos en el año 2017. Se obtuvo una muestra de 105 pacientes. Se evidenció que la mayoría $(86,7 \%)$ de los casos corresponde al sexo masculino con una edad promedio de 33 años. La prevalencia de anemia fue del $13.3 \%$. Las mujeres presentaron más alta prevalencia de anemia. La leucopenia fue más frecuente en el grupo de pacientes con valores de CD4 inferiores 200 cells $/ \mu$. Se concluye que la prevalencia de anemia no fue muy elevada, predominó en el sexo femenino con un patrón normocítico hipocrómico. La leucopenia fue más frecuente en el grupo de pacientes con valores de CD4 inferiores 200 cells/ $\mu$. Las plaquetas en la mayoría de pacientes estuvieron en rango normal.

Palabras claves: Anemia, trombocitopenia, trombocitosis, VIH

\section{Introducción.}

La infección por virus de la inmunodeficiencia humana (VIH) se asocia a numerosas alteraciones hematológicas, observadas en todos los estadios de la enfermedad y de origen multifactorial. Estas alteraciones pueden ocurrir como resultado de la infección por VIH, como secuelas de infecciones relacionadas con el VIH o tumores malignos o como consecuencia de terapias farmacológicas utilizadas para el control de la infección por VIH y otras condiciones asociadas La infección por el VIH está asociada con numerosas anormalidades en la hematopoyesis, que afectan a todas las líneas derivadas de las células pluripotenciales. (1-4)

Dentro de las anormalidades hematológicas de importancia se encuentra las comprometidas con la médula ósea, los elementos celulares de la sangre y la hemostasia. Las causas principales de estas alteraciones se derivan de los efectos directos supresores de la infección del VIH, la hematopoyesis inefectiva, infecciones infiltradas en la médula ósea, deficiencias nutricionales, depuración celular secundaria a esplenomegalia y los efectos de las drogas usadas en el tratamiento de la infección. (5)

Es frecuente encontrar signos de insuficiente maduración celular, como los encontrados en síndromes mielodisplasicos, sinembargo no evolucionan a Leucemia 
La anemia es la citopenia más frecuente, en el momento del diagnóstico se presenta aproximadamente en un $15 \%$, llegando a un $100 \%$ en las etapas finales de la enfermedad. En un estudio prospectivo de una cohorte de 32.867 pacientes, se observó que la incidencia de anemia en un año de seguimiento fue de $37 \%$ en los pacientes con SIDA, $12 \%$ con CD4<200/mm3 y $3 \%$ en pacientes en estadios precoces.(6)

La mayoría de los pacientes presentaron anemia crónica normocrómica normocítica con bajos niveles de reticulocitos y eritropoyetina (EPO) y con bloqueo de la incorporación del hierro. (7) Se ha demostrado que la EPO tiene disminución en la función en los progenitores eritropoyéticos, pese a que se encuentra elevada debido a la respuesta inflamatoria.

Lo importante en el paciente VIH es investigar la causa que produce la anemia, y darle tratamiento individualizado, ya que la anemia es multicausal, y en algunos casos obligaría a cambiar de fármacos instaurados para la terapia Retroviral y de infecciones oportunistas, pudiendo incrementar la mortalidad en los sujetos. Las transfusiones sanguíneas en estados tempranos de la infección podrían estar relacionados en un aumento de la mortalidad, debido a que disminuyen la función de los Linfocitos Natutal Killer y los macrófagos.

Estudios evaluaron en el contexto de la obesidad, las adipocininas secretadas por el tejido adiposo y que están relacionas con los pacientes con VIH, la Leptina está asociada con la masa grasa, que induce a la Hepsidina, que está relacionada con la inflamación y el metabolismo del Hierro.

Otras causas son de origen farmacológico, sobre todo por la Zidovudina, que en los pacientes con SIDA causaba anemias severas en el 20-30\%, actualmente cada vez es menos utilizada.

Las causas gastrointestinales, contribuyen a la deficiencia de hierro de varias maneras, como hemorragia gastrointestinal (shigella, amebiasis, etc.), malabsorción de hierro (giardiasis, cryptosporidiosis) y la intolerancia a la lactosa (en infecciones crónicas). Tumorales por sarcoma de Kaposi y Linfomas, déficit nutricionales (vit B-12 y a. fólico). Las anemias hemolíticas son raras, pueden ser el resultado de hiperesplenismo en pacientes con enfermedades avanzadas del hígado o enfermedades sistémicas. $(4,12)$ Está relacionada con una purpura trombocitopénica trombótica, que es común en algunas infecciones bacterianas.

Aproximadamente en un 15 a 30\% de los pacientes presentan macrocitosis con una dosificación de vitamina B12 y ácido fólico por debajo de valores normales, esta condición probablemente esté relacionada al uso de antirretrovirales y antibióticos, además el transporte de vitaminas y/o una pobre absorción de éstas causadas por una enteropatía de causa infecciosa, pese a que los pacientes consumen altas dosis de ácido fólico. (5-19) 
La leucopenia (leucocitos < 4000/mm3), se presenta la mayoría de las veces en forma concomitante con la anemia. La infección por VIH afecta a todas las células de la serie blanca: neutrófilos, linfocitos y monocitos.

Es habitual encontrar una linfopenia durante los períodos de replicación viral y en las etapas finales de la enfermedad. Principalmente existe un compromiso de los linfocitos CD4, así como de monocitos y macrófagos, lo que determina una alteración en la inmunidad celular, dando como resultado reducción en la capacidad bactericida, proceso de degranulación defectuoso, quimiotaxis anormal, fagocitosis ineficaz.

Es frecuente la neutropenia por dismielopoyesis y por compromiso de las células precursoras por toxicidad farmacológica. En el período de primoinfección puede observarse neutropenia por mecanismo autoinmune (1-4)

La trombocitopenia es una complicación habitual en los pacientes con VIH. En un 50\% cursan asintomáticas, los casos sintomáticos se presentan como un síndrome purpúrico petequial y sangrados mucosos leves. Otras causas de trombocitopenia de causa infecciosa son provocadas por las enfermedades oportunistas como histoplasmosis y criptococosis. Fármacos utilizados en el manejo de infecciones como el Ganciclovir, Pentanidina y Clotrimazol están asociados a trombocitopenia. En estadios avanzados de la enfermedad la presencia de organomegalias y adenopatías asociados a trombocitopenia sugieren la presencia de linfoma no Hodgkin.

Los megacariocitos son destruidos durante la replicación viral, observándose a nivel del aspirado una médula amegacariocítica, siendo esta una manifestación de una trombocitopenia de origen central.

Cuando existe destrucción incrementada de las plaquetas, si se realiza un estudio de aspirado medular, se visualiza un aumento en el número de megacariocitos. Una teoría sostiene que los complejos inmunes circulantes son depositados en la membrana plaquetaria y que son destruidos por las células reticuloendotelial. Se cree que estos complejos inmunes contienen anti-HIVgp120 y anti-anticuerpos. $(4,20)$.

En un estudio realizado en una población hispana con infección por VIH, la prevalencia de anemia fue de $41.5 \%$ y fue un predictor importante de mortalidad en un año: $30.8 \%$ en la severa, $23.3 \%$ en la moderada, $8.4 \%$ leve y $2.5 \%$ sin anemia.(8) Estudios realizados en el Ecuador sobre la prevalencia de anemia en pacientes sin infección por VIH muestran que las mujeres en edad reproductiva presentan una prevalencia del 8.5\%.(21) Dado que existe un incremento de casos de infección por VIH en este grupo poblacional es importante caracterizar la presentación de la anemia y las otras citopenias como leucopenia y trombocitopenia. 
ISSN: 2600-5859

El objetivo de este estudio retrospectivo transversal es evaluar la frecuencia de anomalías hematológicas más frecuentes en pacientes portadores del virus de la inmunodeficiencia humana, atendidos en el Hospital Enrique Garcés Quito Ecuador.

\section{Metodología.}

Tipo de estudio: Se trata de un estudio descriptivo, retrospectivo.

Tipo de muestreo: muestreo aleatorio simple

Tamaño de la muestra: 105 pacientes, se aplicó la fórmula para muestreo aleatorio simple para un universo finito: 1200 pacientes con diagnóstico de infección con VIH atendidos en el año 2017. Donde:

$$
\begin{aligned}
& \mathrm{N}=\text { Total de la población } 1200 \\
& \mathrm{Za} 2=1.962(\text { si la seguridad es del } 95 \%) \\
& \mathrm{p}=\text { proporción esperada }(\text { en este caso } 45 \%=0.45) \\
& \mathrm{q}=1-\mathrm{p}(\text { en este caso } 1-0.45=0.65) \\
& \mathrm{d}=\text { precisión }(\text { en este caso deseamos un } 3 \%) .
\end{aligned}
$$

Recolección de la información: Se realizó mediante la revisión de historias clínicas.

Procesamiento y análisis de los datos: Se realizó utilizando el programa SPSS 22.

\section{PACIENTES:}

Universo: 1200 pacientes registrados en las historias clínicas de la Unidad de atención integral de VIH-SIDA del Hospital Dr. Enrique Garcés. Se tomará una muestra 105 pacientes, cálculo realizado para muestreo aleatorio simple.

Unidad de estudio: Unidad de atención integral de VIH-SIDA del Hospital Enrique Garcés, en Quito - Ecuador.

Criterios de inclusión: Todos los pacientes con diagnóstico de VIH, acuden a controles periódicos.

\section{Resultados}

\section{Descriptores de la población estudiada.}

Se estudió una muestra de 105 casos atendidos en el año 2017, en la unidad de atención integral a VIH-SIDA.

Tabla 1. Sexo de los pacientes con VIH

\begin{tabular}{lll}
\hline Sexo & Frecuencia $(\mathbf{n})$ & Porcentaje $(\boldsymbol{\%})$ \\
\hline Masculino & 91 & 86,7 \\
Femenino & 14 & 13,33 \\
\hline
\end{tabular}


ISSN: 2600-5859

Tabla 2. Niveles CD4 de los pacientes con VIH

\begin{tabular}{llcc}
\hline & Nivel de CD4 & Frecuencia & Porcentaje \\
\hline 1$) \quad \geq 500$ cells $/ \mu \mathrm{L}$ & 13 & 12,4 \\
2) & $200-499$ cells $/ \mu \mathrm{L}$ & 51 & 48,6 \\
$3) \quad<200$ cells $/ \mu \mathrm{L}$ & 41 & 39 \\
Total & $\mathbf{1 0 5}$ & $\mathbf{1 0 0}$ \\
\hline
\end{tabular}

El valor de los CD4 en promedio fue de 135 cells $/ \mu \mathrm{L}$ y el $48,6 \%$ de los pacientes se encontraban en la categoría 2 de la CDC (200-499 cells/ $\mu \mathrm{L})$.

Tabla 3. Leucocitos de los pacientes con VIH

\begin{tabular}{|c|c|c|c|c|c|c|}
\hline & $\begin{array}{l}\text { Leucocito } \\
\left(10^{3} / \mu \mathrm{L}\right)\end{array}$ & $\begin{array}{l}\text { seutrofilos } \\
\left(10^{3} / \mu \mathrm{L}\right)\end{array}$ & $\begin{array}{l}\text { Linfocitos } \\
\left(10^{3} / \mu \mathrm{L}\right)\end{array}$ & $\begin{array}{l}\text { Monocitos } \\
\left(10^{3} / \mu \mathrm{L}\right)\end{array}$ & $\begin{array}{l}\text { Eosinofilos } \\
\left(10^{3} / \mu \mathrm{L}\right)\end{array}$ & $\begin{array}{l}\text { Basófilos } \\
\left(10^{3} / \mu \mathrm{L}\right)\end{array}$ \\
\hline Media & 454,90 & 257,97 & 183,19 & 42,33 & 16,79 & 2,98 \\
\hline Mediana & 463,00 & 248,00 & 169,00 & 43,00 & 10,00 & 2,50 \\
\hline Desviación estándar & 252,931 & 147,504 & 146,367 & 17,444 & 23,398 & 1,678 \\
\hline Varianza & 63973,95 & 21757,37 & 21423,38 & 304,30 & 547,450 & 2,815 \\
\hline
\end{tabular}

Tabla 4. Índices hematimétricos de los pacientes con VIH

\begin{tabular}{lccccccc}
\hline & $\begin{array}{l}\text { Hemo- } \\
\text { globina } \\
(\mathrm{g} / \mathrm{dl})\end{array}$ & $\begin{array}{l}\text { Hemato- } \\
\text { crito }(\%)\end{array}$ & $\begin{array}{l}\text { VCM } \\
(\mathrm{fL})\end{array}$ & $\begin{array}{l}\mathrm{MCH} \\
(\mathrm{pg})\end{array}$ & $\begin{array}{l}\text { MCHC } \\
(\mathrm{g} / \mathrm{dl})\end{array}$ & $\begin{array}{l}\text { PLAQUETAS } \\
\left(10^{3} / \mu \mathrm{L}\right)\end{array}$ & $\begin{array}{l}\text { VM PLAQUE- } \\
\text { TARIO(fL) }\end{array}$ \\
\hline Media & $15,3,56$ & $44,1,61$ & 126 & 25,6 & 31,01 & 271,92 & 97,77 \\
Mediana & $15,6,00$ & $45,7,00$ & 88,1 & 30,2 & 33,9 & 259 & 96 \\
$\begin{array}{l}\text { Desviación } \\
\text { estándar }\end{array}$ & 11,65 & 49,78 & 19,2 & 10,6 & 9,63 & 71,172 & 84,258 \\
Varianza & 135,75 & 247,817 & 3669 & 112 & 92,75 & 50,65 & 70,99 \\
\hline
\end{tabular}

$\mathrm{MCV}=$ volumen celular medio, $\mathrm{MCH}=$ hemoglobina celular media, $\mathrm{MCHC}=$ concentración media de hemoglobina celular

En la biometría la media de la hemoglobina y las plaquetas indican que está dentro de parámetros normales, en cambio los leucocitos y linfocitos tiene una media en niveles bajos. 
ISSN: 2600-5859

Tabla 5. Parámetros hematológicos de la población del estudio estratificado por los valores del contaje de Linfocitos CD4 cell $/ \mathrm{mm}^{3}$

\begin{tabular}{|c|c|c|c|c|c|}
\hline \multirow[t]{2}{*}{ Parámetro } & \multirow[b]{2}{*}{ estadio $1(>500)$} & \multicolumn{4}{|c|}{ Contaje de Linfocitos CD4 cell/mm3 } \\
\hline & & estadio 2(200-499) & estadio $3(<200)$ & $\mathbf{F}$ & valor $p$ \\
\hline Edad (años) & $28,45+9,35$ & $29,90+9,15$ & $31,00+9,60$ & 0,396 & 0,674 \\
\hline \multicolumn{6}{|c|}{ Parámetros hematológicos } \\
\hline Hemoglobina (g/dL) & $16.5+0.70$ & $16.66+1.366$ & 17 & 0,051 & 0,951 \\
\hline Hematocrito (\%) & 49 & $45,83+4,98$ & $41.5+2.12$ & & \\
\hline $\mathrm{MCV}(\mathrm{fL})$ & $84+2.82$ & $88.33+3.55$ & $78,00+1412$ & 2.12 & 0.18 \\
\hline $\mathrm{MCH}(\mathrm{pg})$ & 26 & $30,43+2,065$ & $26,33+2,517$ & 5.992 & 0.012 \\
\hline $\mathrm{MCHC}(\mathrm{g} / \mathrm{dL})$ & 32 & $34,17+0,98$ & $33,00+1,155$ & 2.669 & 0.129 \\
\hline Plaquetas $(103 / \mu \mathrm{L})$ & $274.65+71.86$ & $266.2+72.154$ & $285.59+68.87$ & 0.613 & 0.544 \\
\hline Leucocitos & $4264+302.28$ & $4264.28+227.76$ & $5375.2+277,28$ & 1.723 & 0.184 \\
\hline Neutrofilos & $232.95+96.22$ & $242,16+142,178$ & $311,83+191,302$ & 3.068 & 0.083 \\
\hline Linfocitos & $232,95+96,221$ & $246,06+139,890$ & $311,83+191,302$ & 2.064 & 0.132 \\
\hline Monocitos & $226,85+239,153$ & $175,76+101,082$ & $165,22+146,079$ & 1.146 & 0.322 \\
\hline Eosinofilos & $37,75+17,669$ & $42,02+17,127$ & $47,17+17,655$ & 1.605 & 0.206 \\
\hline Basofilos & $16,75+13,038$ & $17,85+27,672$ & $13,82+17,159$ & 0.238 & 0.789 \\
\hline
\end{tabular}

Los valores de $\mathrm{P}<0.05$ se consideraron significativos. Los valores $\mathrm{P}$ representan ANOVA de un factor de todas las variables. $\mathrm{MCV}=$ volumen celular medio, $\mathrm{MCH}=$ hemoglobina celular media, $\mathrm{MCHC}=$ concentración media de hemoglobina celular,

\section{Análisis Multivariable}

Se realizó un análisis multivariable comparando las diferencias de las medias, utilizando el ANOVA de un factor, se tomó en cuenta los valores de los parámetros de la biometría en relación los niveles de contaje de Linfocitos CD4 cell $/ \mathrm{mm}^{3}$, se obtuvo la media y la desviación estándar de cada parámetro de la biometría y se obtuvo el valor de la diferencia del ANOVA de frecuencias $(\mathrm{F})$ y el valor de $\mathrm{p}$.

No existieron diferencias significativas entre los niveles de contaje de Linfocitos CD4 cell/ $/ \mathrm{mm}^{3}$. Estadio 1(>500), Estadio 2(200-499), Estadio 3(<200) con las variables de los parámetros de la biometría hemática excepto con la hemoglobina corpuscular media que presentó diferencia estadísticamente significativa, con una $\mathrm{p}<0.05$. 
Del total de los 1200 pacientes registrados en las historias clínicas de la Unidad de atención integral de VIH-SIDA del Hospital Dr. Enrique Garcés, se obtuvo, por muestreo aleatorio simple, una muestra 105 pacientes.

La mayoría de los casos corresponden al sexo masculino, con una edad promedio de 33 años; predominó la orientación sexual: hombres que tienen sexo con hombres.

El tratamiento que recibían la mayoría de pacientes era el esquema Tenofovir Emtricintavina y Efavirenz, sólo un paciente recibía Zidovudina. Se utilizó Trimetoprin-Sulfametoxazol para profilaxis de infección por Pneumocystis Jirovecii en aquellos pacientes en los cuales los niveles de linfocitos CD4 fueron inferiores a 200 cells $/ \mu \mathrm{L}$.

En el recuento de las células CD4 influyen varios factores independientes a la infección por VIH. Ciertos medicamentos o infecciones, que están asociados con la leucopenia, pueden provocar la depresión del recuento absoluto de células CD4. ( 6 ) Por el contrario, medicamentos específicos o infecciones, que conducen a la leucocitosis, pueden dar lugar a recuentos elevados de células CD4. (1-3). Por este motivo, es interesante tener siempre en cuenta los valores relativos de CD4 (\%), ya que estos se ven menos alterados por las fluctuaciones de la cifra total de leucocitos. En el estudio se encontró que las células CD4 fueron en promedio 136 cells/ $\mu \mathrm{L}$ y un $39 \%$ se encontraba en la categoría 3 del CDC $(<200$ cells $/ \mu \mathrm{L})$, lo que implica en este grupo una alta susceptibilidad a las infecciones oportunistas. El porcentaje de linfocitos CD4 en promedio fue del $15.3 \%$ en todo el estudio y con valores de CD4 $<200$ cells/Ml, lo que representa un 11.08 $\%$.

La prevalencia de anemia en el estudio se definió con una concentración de hemoglobina menor de $12 \mathrm{~g} / \mathrm{dl}$ en las mujeres y menor de $14 \mathrm{~g} / \mathrm{dl}$ en los varones, evidenciándose una prevalencia del $13.3 \%$. Probablemente esta baja prevalencia comparada con otros estudios se deba a múltiples factores, el más importante dado por la altura de la ciudad de Quito $(2.850 \mathrm{~m})$, la buena adherencia al tratamiento antirretroviral, y la alta prevalencia de enfermedades pulmonares en la zona, que producen poliglobulia. (21). También destaca el hecho de que sólo uno de los 105 pacientes tenía en su tratamiento con Zidovudina. En otros estudios se encontró que la anemia era más prevalente en personas de mayor edad, que además presentaba bajo índice de masa corporal y bajos niveles de contaje CD4. $(2,3)$

En relación al contaje de CD4 y anemia, en los niveles de CD4 menores de 500 cells $/ \mu \mathrm{L}$ se encontró mayor prevalencia de anemia. Las mujeres presentaron más alta prevalencia de anemia que los hombres, en concordancia con otros estudios en los cuales se demostró una alta prevalencia de anemia en mujeres jóvenes.

Con respecto al volumen corpuscular medio (VCM) se encontró en el estudio que la mayoría de pacientes con anemia tenía el VCM normal con tendencia similar a otros estudios publicados. Un 
ISSN: 2600-5859

estudio valoró los parámetros del estado del hierro y encontró que no había deficiencia de hierro o sobrecarga de hierro en los grupos con VIH /SIDA, un gran porcentaje de pacientes tenía anemia de enfermedad crónica. La anemia es, generalmente, normocítica hipocrómica en pacientes con SIDA. (24)

La leucopenia fue más frecuente en el grupo de pacientes con valores de CD4 de 200 cells/ $\mu \mathrm{L}$, estos hallazgos son concordantes con otros estudios publicados. (1)

Muy pocos pacientes presentaron trombocitopenia, no hubo relación con los niveles de CD4; similares hallazgos fueron encontrados en otros estudios. Estos hallazgos sugieren que los niveles de plaquetas no son un buen marcador de progresión de la enfermedad. Además, la trombocitopenia puede presentarse como una de las primeras manifestaciones clínicas, y no denota inmunodeficiencia. (1-5)

\section{Conclusiones.}

- Las alteraciones hematológicas en pacientes con VIH, son frecuentes y variadas, ya que los pacientes presentan una amplia gama de enfermedades infecciosas y no infecciosas.

- Los linfocitos CD4 en los pacientes de estudio estuvieron en promedio 136 cells/ $\mu \mathrm{L}$, esto debido a que la mayoría de sujetos fueron recién diagnosticados, por lo que la enfermedad no estaría bien controlada, lo que llevaría a incrementar el riesgo de sufrir infecciones oportunistas.

- La anemia es una complicación frecuente del VIH, con una prevalencia del $13.3 \%$. Las mujeres presentaron más alta prevalencia de anemia que los hombres, por lo que resulta importante buscar la causa de la misma y darle el tratamiento etiológico necesario.

- La mayoría de los pacientes con anemia tenía el Volumen Corpuscular Medio normal y la Concentración de hemoglobina corpuscular media disminuida, presentando una anemia normocítica, hipocrómica (que caracteriza a la anemia inflamatoria de la enfermedad crónica).

- La leucopenia fue más frecuente en el grupo de pacientes con valores de CD4 por debajo de 200 cells/ $\mu \mathrm{L}$.

- La severidad de la anemia y leucopenia están en relación con el contaje bajo de Linfocitos CD4.

- La mayoría de paciente presenta valores normales de plaquetas incluyendo los pacientes con contaje de CD4 menor de 200 cells $/ \mu \mathrm{L}$.

\section{Referencias bibliográficas.}

1. Sloand E1.. Hematologic complications of HIV infection. AIDS Rev. 2005 OctDec;7(4):187-96.

2. Opie J. Haematological complications of HIV infection.S Afr Med J 2012;102(6):465-468.

3. De Santi G, Menezes D. Hematological abnormalities in HIV-infected patients. International Journal of Infectious Diseases 15 (2011) e808-e811. 
4. Mehta S . Jutur S. Gautam D. Hematologic Manifestations of HIV/AIDS. Medicine Update-2011; 483:490

5. Wilfred D. Magnitude and correlates of moderate to severe anemia among adult HIV patients receiving first line HAART in Northwestern Tanzania: a cross sectional clinic based study. Pan African Medical Journal. 2016; 23:26

6. Lai JL, Chen YH, Liu YM, Yuan J, Prevalence and risk factors of anemia in hospitalised HIV- infected patients in southeast China. Epidemiology Infected 2019, 235-242.

7. Wisaksana et al. Anemia and iron homeostasis in a cohort of HIVinfected patients in Indonesia BMC Infectious Diseases 2011, 11:213

8. Santiago-Rodríguez et al. Anemia in a cohort of HIV-infected Hispanics: prevalence, associated factors and impact on one-year mortalityBMC Research Notes 2014, 7:439

9. Pande A, Bhattacharyya M, Pain S, Ghosh B, Saha S, Ghosh A, Banerjee A. Anemia in Antiretroviral Naïve HIV/AIDS Patients: A Study from Eastern India. Online J Health Allied Scs. 2011;10(4):4

10. Meidani, et al.: Prevalence, severity, and related factors of Anemia in HIV/AIDS patients. Journal of Research in Medical Sciences 2012; 17(2): 138-42.

11. Freire W.B., Ramírez MJ., Belmont P., Mendieta MJ., Silva MK., Romero N., Sáenz K., Piñeiros P., Gómez LF., Monge R. 2013. Encuesta Nacional de Salud y Nutrición del Ecuador. ENSANUT-ECU 2011-2013 MSP.INECQuito, Ecuador.

12. Sanz K., Valores de referencia hematológicos en población alto andina ecuatoriana, empleando analizador SYSMEX XE-2100®* Rev Mex Patol Clin 2008; 55: 207-215.

13. Ogbe PJ1, Idoko OA, Evaluation of iron status in anemia of chronic disease among patients with HIV infection Clin Lab Sci. 2012 Winter;25(1):7-12.

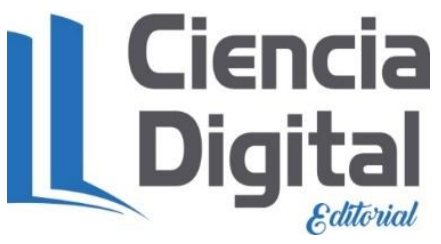




\section{Para citar el artículo indexado}

Pacheco Toro, S., Fierro Vasco, S., Vega Vasco, J., \& Vega Vasco, S. (2020). Alteraciones hematológicas en pacientes portadores del virus de lnmunodeficiencia Humana. ConcienciaDigital, 3(1.1), 253-263. https://doi.org/10.33262/concienciadigital.v3i1.1.1145

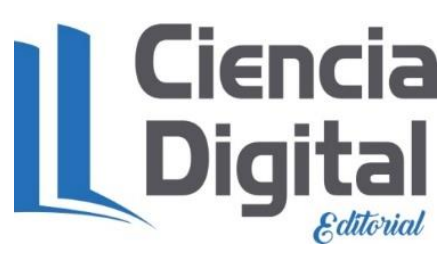

El artículo que se publica es de exclusiva responsabilidad de los autores y no necesariamente reflejan el pensamiento de la Revista Conciencia Digital.

El articulo queda en propiedad de la revista y, por tanto, su publicación parcial y/o total en otro medio tiene que ser autorizado por el director de la Revista Conciencia Digital.
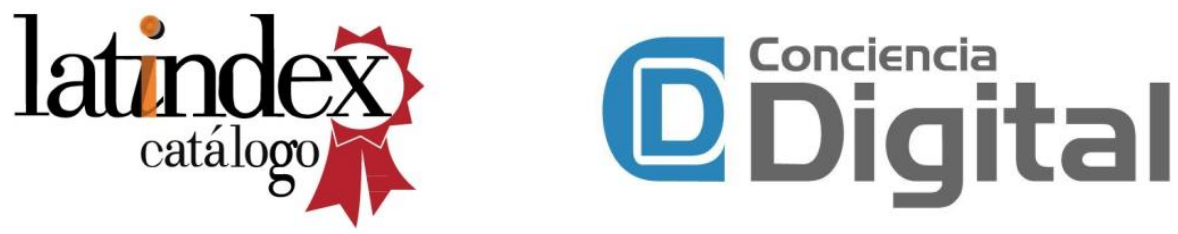